\section{Comparison of left versus right lateral starting position on colonoscopy: a systematic review and meta-analysis of randomized controlled trials}

\author{
Jun Watanabe ${ }^{a, b}$ \\ Jichi Medical University, Shimotsuke-City, Tochigi, Japan
}

We read the article by Ramai et al [1] with great interest, and we appreciate the authors' efforts to assess the efficacy of a right lateral starting position on colonoscopy compared to a left lateral starting position. However, we would like to point out 2 concerns.

First, the authors should follow the Preferred Reporting items for Systematic Review and Meta-Analyses (PRISMA) guidelines [2]. The author should provide details of the predefined protocol and summarize the strength of the evidence for each major result using the Grades of Recommendation, Assessment, Development, and Evaluation (GRADE) approach [3]. We evaluated that the certainty of the evidence regarding the rate of cecal intubation and cecal intubation time was moderate, because of imprecision due to the small sample size for the rate of cecal intubation, and low, because of imprecision and risk of bias due to skewed distribution, respectively. The author should state their conclusions, including the strength of evidence.

Second, this was not a first systematic review but an updated review. The previous systematic reviews showed that, similar to the results of their study, right lateral position did not reduce cecal insertion time as in this study, but the supine (mean difference [MD] $-41.0 \mathrm{sec}, 95 \%$ confidence interval $[95 \% \mathrm{CI}]-57.3$ to -24.7$)$ and tilt-down position

\section{Authors' reply}

\section{Daryl Ramai ${ }^{a}$, Douglas G. Adler ${ }^{a, b}$ \\ University of Utah, Salt Lake City; Porter Adventist Hospital/PEAK Gastroenterology, Denver, Colorado, USA}

We thank Jun Watanabe for his interest in our study [1]. As stated in our protocol, our study followed the Preferred Reporting items for Systematic Review and Meta-Analyses (PRISMA) guidelines [2]. We predefined our study hypothesis and carried out a detailed systematic search with the assistance of an expert librarian. Since our meta-analysis involved only randomized clinical trials (RCTs), we used the Jadad scale to assess the quality of these studies [3]. To assess the quality of evidence, the Grades of Recommendation, Assessment, Development, and Evaluation (GRADE) approach is recommended [4].
(MD -37.3 sec, 95\%CI -72.1 to -2.4 ) reduced mean cecal insertion time for colonoscopy compared with the left lateral position $[4,5]$.

\section{References}

1. Ramai D, Singh J, Brooks OW, et al. Comparison of left versus right lateral starting position on colonoscopy: a systematic review and meta-analysis of randomized controlled trials. Ann Gastroenterol 2021;34:699-704.

2. Moher D, Shamseer L, Clarke M, et al; PRISMA-P Group. Preferred reporting items for systematic review and meta-analysis protocols (PRISMA-P) 2015 statement. Syst Rev 2015;4:1.

3. Guyatt G, Oxman AD, Akl EA, et al. GRADE guidelines: 1. Introduction-GRADE evidence profiles and summary of findings tables. J Clin Epidemiol 2011;64:383-394.

4. Watanabe J, Park D, Kakehi E, Inoue K, Ishikawa S, Kataoka Y. Efficacy and safety of the starting position during colonoscopy: a systematic review and meta-analysis. Endosc Int Open 2020;8:E848-E860.

5. Lin SY, Yaow CYL, Ng CH, Wong NW, Tham HY, Chong CS. Different position from traditional left lateral for colonoscopy? A meta-analysis and systematic review of randomized control trials. Chronic Dis Transl Med 2021;7:27-34.

${ }^{a}$ Department of Surgery, Division of Gastroenterological, General and Transplant Surgery; ${ }^{\mathrm{b} D i v i s i o n}$ of Community and Family Medicine, Jichi Medical University, Shimotsuke-City, Tochigi, Japan

Conflict of Interest: None

Correspondence to: Jun Watanabe, $\mathrm{MD}, \mathrm{PhD}$, Division of Community and Family Medicine, Jichi Medical University, 3311-1 Yakushiji,

Shimotsuke City, Tochigi, 329-0498, Japan, e-mail: m06105jw@jichi.ac.jp

Received 12 June 2021; accepted 13 July 2021;

published online 14 September 2021

DOI: https://doi.org/10.20524/aog.2021.0678

In this approach, direct evidence from RCTs starts at high quality and can be rated down based on risk of bias, indirectness, imprecision, inconsistency (or heterogeneity) and/or publication bias, to levels of moderate, low, and very low quality. According to our analysis of the quality of the evidence, while there was no evidence of publication bias, the evidence was rated down for inconsistency, imprecision, and the risk of bias due to the unblinded nature of right and left lateral colonoscopy trials. Therefore, we concluded that while our meta-analysis shows no difference in right vs. left lateral colonoscopy with regard to the rate of cecal intubation or cecal intubation time, this is based on a low quality of evidence.

To date, our study included the largest cohort of patients in which right and left lateral positions for colonoscopy were compared specifically. We appreciate the author drawing our attention to other positions (i.e., supine, tilt-down, etc.) and referencing his study, which attempted to use meta-analytic tools to compare 4 different positions for colonoscopy [5]. However, in this study each position had only $2-3$ studies for 
pooled analysis. This raises a question regarding the necessity of performing meta-analyses with very small sample sizes.

\section{References}

1. Ramai D, Singh J, Brooks OW, et al. Comparison of left versus right lateral starting position on colonoscopy: a systematic review and meta-analysis of randomized controlled trials. Ann Gastroenterol 2021;34:699-704.

2. Moher D, Shamseer L, Clarke M, et al; PRISMA-P Group. Preferred reporting items for systematic review and meta-analysis protocols (PRISMA-P) 2015 statement. Syst Rev 2015;4:1.

3. Jadad AR, Moore RA, Carroll D, et al. Assessing the quality of reports of randomized clinical trials: is blinding necessary? Control Clin Trials 1996;17:1-12.

4. Guyatt G, Oxman AD, Akl EA, et al. GRADE guidelines: 1. Introduction-GRADE evidence profiles and summary of findings tables. J Clin Epidemiol 2011;64:383-394.

5. Watanabe J, Park D, Kakehi E, Inoue K, Ishikawa S, Kataoka Y. Efficacy and safety of the starting position during colonoscopy: a systematic review and meta-analysis. Endosc Int Open 2020;8:E848-E860.

aDivision of Gastroenterology and Hepatology, University of Utah,

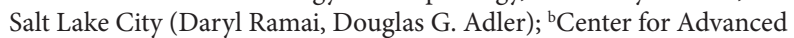
Therapeutic Endoscopy (CATE), Porter Adventist Hospital/PEAK Gastroenterology, Denver, Colorado (Douglas G. Adler), USA

Conflict of Interest: None

Correspondence to: Douglas Adler, MD. Center for Advanced Therapeutic Endoscopy (CATE), Porter Adventist Hospital, Denver, Colorado, USA, e-mail: dougraham2001@gmail.com

Received 7 July 2021; accepted 13 July 2021; published online 14 September 2021

DOI: https://doi.org/10.20524/aog.2021.0659 